Article:

\title{
Adaptive coatings based on polyaniline for direct 2D observation of diffusion processes in microfluidic systems
}

Larisa Florea, Alain Martin-Mayor, M. Mounir Bou-Ali, Kate Meagher, Dermot Diamond, Mustafa Tutar, Fernando Benito-López

Sensors and Actuators B: Chemical 231 : 744-751 (2016)

This work is made available online in accordance with publisher policies. To see the final version of this work please visit the publisher's website. Access to the published online version may require a subscription.

\section{Link to publisher's version:}

http://dx.doi.org/10.1016/j.snb.2016.03.085

Copyright statement: (C) 2016 Elsevier Ltd. Full-text reproduced in accordance with the publisher's self-archiving policy.

This manuscript version is made available under the CC-BY-NC-ND 4.0 license http://creativecommons.org/licenses/by-nc-nd/4.0

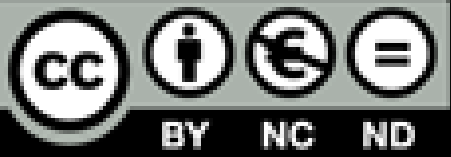




\title{
ADAPTIVE COATINGS BASED ON POLYANILINE \\ FOR DIRECT 2D OBSERVATION OF DIFFUSION PROCESSES \\ IN MICROFLUIDIC SYSTEMS
}

\author{
Larisa Florea ${ }^{1, *}$, Alain Martin-Mayor ${ }^{2}$, M. Mounir Bou-Ali, ${ }^{2}$ Kate Meagher ${ }^{1}$, \\ Dermot Diamond ${ }^{1}$, Mustafa Tutar ${ }^{2,3}$, Fernando Benito-Lope $z^{* 1,4}$ \\ ${ }^{I}$ Insight: Centre for Data Analytics, National Centre for Sensor Research, Dublin City University, \\ Dublin, Ireland \\ ${ }^{2}$ Mondragon Unibertsitatea, Arrasate-Mondragón, Spain \\ ${ }^{3}$ Ikerbasque, Basque Foundation for Science, Bilbao, Spain \\ ${ }^{4}$ Microfluidics UPV/EHU Cluster, Analytical Chemistry Department, University of the Basque \\ Country UPV/EHU, Vitoria-Gasteiz, Spain
}

\begin{abstract}
Microfluidic devices are poised to dramatically influence the future of the process industry. Therefore the understanding and proper evaluation of the flow and mixing behaviour at microscale becomes a very important issue. In this study, the diffusion behaviour of two reacting solutions of $\mathrm{HCl}$ and $\mathrm{NaOH}$ were directly observed in a glass/polydimethylsiloxane microfluidic device using adaptive coatings based on the conductive polymer polyaniline that are covalently attached to the microchannel walls. The two liquid streams were combined at the junction of a Y-shaped microchannel, and allowed to diffuse into each other and react. The results showed excellent correlation between optical observation of the diffusion process and the numerical results. A numerical model which is based on finite volume method (FVM) discretisation of steady Navier-Stokes (fluid flow) equations and mass transport equations without reactions was used to calculate the flow variables at discrete points in the finite volume mesh element. The high correlation between theory and practical data indicates the potential of such coatings to monitor diffusion processes and mixing behaviour inside microfluidic channels in a dye free environment.
\end{abstract}


Keywords: Polyaniline; Mixing process; Adaptive coatings; Optical sensor; Microfluidics; Computational fluid dynamics (CFD);

\section{Introduction}

Lab-on-a-chip technology is attracting increasing interest as the miniaturisation of reaction systems offers significant practical advantages over classical bench-top chemical synthesis, such as smaller volumes of reagents, shorter reaction times, low energy consumption, flexible control of reaction conditions and reduced costs. In particular, rapid mixing of the fluids flowing through a microchannel is very important for various applications of microfluidic systems, including biochemical processes like polymerase chain reaction (PCR), DNA purification, sequencing or synthesis of nucleic acids and chemical reactions, among others[1, 2].

Consequently, a number of microfluidic device designs have been produced to create rapid mixing conditions. These comprise active mixers that have mechanical moving parts or use external fields to agitate the fluid[3, 4] and passive mixers that comprise different design geometries of the microfluidic channel in order to increase the interfacial surface area between initially distinct fluid regions $[5,6]$.

In addition, on-chip detection techniques are essential for the continuous monitoring of the mixing behaviour of confluent streams. For this purpose many spectroscopic detection methods have been employed such as laser-induced fluorescence[7-9], confocal fluorescence microscopy[10], ultraviolet absorption[11, 12], chemiluminescence[13, 14], and confocal Raman microscopy[10]. These spectroscopic techniques provide good opportunities for the detection of chemical species and are suitable for studying mixing in microfluidic devices. However, they typically require the addition of a dye or pretreatment of a solute species with florescent tags to allow on-chip detection[9, 15]. Consequently, in these approaches one 
follows the bulk behaviour of a solute added solely to visualise diffusion and reaction processes, rather than the solvent/liquid itself. The continuing growing trends of microfluidics highlight the importance of understanding the mechanisms and fundamental differences involved in microscale fluid flow and mixing at microscale. With rapid developments in computational power and memory size, numerical methods in the context of computational fluid dynamics (CFD) have become useful tools to resolve diffusion process in microfluidic structures in more realistic manner through integration of new elaborated numerical techniques. This new branch of fluid dynamics complements experimental and theoretical fluid dynamics by providing an alternative cost-effective means of simulating real flows and demonstrates the ability of tuning of various microfluidic parameters to optimise diffusion performance[16].

Several adaptive coatings have been developed by us and others that can be used for dynamic detection of the fluid passing inside the microcapillary/microchannel, such as polyaniline coatings for $\mathrm{pH}[17]$, ammonia[18], and L-ascorbic acid detection[19], photochromic coatings for solvent sensing[20] and metal ion sensing[21], ionophore containing coatings for calcium ions detection or Fluorescein based coatings for $\mathrm{pH}$ sensing[22].

Although the potential of these coatings can be easily appreciated, very few examples are presented so far in the literature. We believe that stimuli-responsive polymeric coatings grafted on the inner walls of a microchannel or microcapillary, that change their optical proprieties in response to the analyte passing inside, offer the possibility of building platforms in which the microfluidic system itself becomes an optical sensor. Having an inherently responsive microchannel or microcapillary means that specific analytes moving through the system can be detected dynamically along the entire channel length in real time, without the need to add reagents to the sample. On this basis, we have recently developed polyaniline based coatings grafted to the inner walls of a microfluidic channel that can be 
used for dynamic mapping of $\mathrm{pH}$ gradients using digital imaging, along the entire channel length in real time[17].

At an acidic $\mathrm{pH}$, polyaniline exists in its doped conductive state, the emeraldine salt (ES). The ES may be converted to the corresponding emeraldine base (EB) by treatment with an alkali solution. Imine sites of the EB forms are easily protonated, with a striking insulatorconductor transition, induced due to the appearance of polarons in the lattice, while the number of p-electrons remains constant. As a consequence, new optical properties appear in the ES[23]. Thus, PAni has huge potential for sensing applications and has extensively been used as material for optical $\mathrm{pH}$ sensors due to its strong $\mathrm{pH}$ sensitivity[17, 24-26]. This propriety indicates that coatings based on polyaniline could be used to study mixing in fluidic devices when merging solutions of different $\mathrm{pH}$.

In the present study, these adaptive coatings have been employed to monitor the diffusion and mixing processes of two reactive fluids $(\mathrm{HCl}$ and $\mathrm{NaOH})$ after converging in the microchannel. The processes have been characterised experimentally and validated numerically, with excellent correlation of the mixing point location under differing flow conditions.

\section{Experimental}

\subsection{Materials and methods}

Aniline (BDH), $\mathrm{HCl}$ (Fisher Scientific), ammonium persulfate (Aldrich), $\mathrm{N}$-[3(Trimethoxylsilyl) propyl] aniline (Aldrich), sodium hydroxide (Aldrich) were used. The aniline monomer was purified by vacuum distillation before use. Other chemicals were used as received.

$\mathrm{UV}-\mathrm{Vis}$ Spectroscopy was used to study the $\mathrm{pH}$ dependence of the polyaniline coatings. The absorbance spectra were recorded using two fiber-optic light guides connected to a Miniature 
Fiber Optic Spectrometer (USB4000 - Ocean Optics) and aligned using an in-house made cell (see Figure S2). The light source used was a Halogen light source (Top Sensor Systems).

\subsection{Microfluidic device fabrication}

The fabrication of the master mold was carried out using a laser ablation system-excimer/ $\mathrm{CO}_{2}$ laser (Optec Laser Micromachining Systems, Belgium) by cutting the microfluidic structures in a 2x50 $\mu \mathrm{m}$ double-sided pressure sensitive adhesive film, PSA, (AR8890, Adhesives Research, Ireland) and pasting one of the PSA sides to a petri dish[15].

For PDMS casting, the precursor was prepared by mixing PDMS elastomer with the curing agent from Sylgard 184 kit at a weight ratio of 10:1, poured onto a master mould, and cured in an oven at $80{ }^{\circ} \mathrm{C}$ for $2 \mathrm{~h}$. Following curing, the PDMS layer is peeled from the master. The inlets and outlets $(800 \mu \mathrm{m}$ in diameter) were made using a manual puncher (Technical Innovations, Inc., Brazoria, TX). The surfaces of the two pieces - PDMS and glass (35 $\mathrm{mm} \mathrm{x}$ $64 \mathrm{~mm}$, Agar Scientific Limited, England) were cleaned and exposed to oxygen plasma. Following this, the two pieces of PDMS and glass were immediately brought into conformal contact to form an irreversible seal. Silicon tubes were employed to further connect the main inlets with a syringe pump (PHD 2000 Syringe, Harvard Apparatus) for sample delivery and washing.

\subsection{Microfluidic device functionalisation}

The functionalisation of the inner walls of the microfluidic channel with PAni nanofibres was achieved using a previous procedure[17]. Briefly, the PDMS layers containing the microfluidic channel $(1000 \mu \mathrm{m} \times 100 \mu \mathrm{m})$ and the glass slides were exposed to oxygen plasma for $60 \mathrm{~s}$ (Harrick Plasma) and put together to form an irreversible seal. Following this, $20 \%$ wt solution of $\mathrm{N}$-[3- (trimethoxylsilyl) propyl]aniline in ethanol was passed through 
the channel at a flow rate $(Q)$ of $0.5 \mu \mathrm{L} \min ^{-1}$ for $60 \mathrm{~min}$. This technique ensured the formation of a silane-bearing aniline monolayer on the PDMS (glass) substrates via molecular self-assembly that could serve as one of the initiation site for PAni polymerisation and was also used to covalently anchor the PAni chain on the substrate[27]. Coating of PAni nanofibres on the micro-channel was performed by filling the microchannel with freshly prepared $1 \mathrm{M} \mathrm{HCl}$ solution containing ammonium peroxydisulfate and aniline in a molar ratio of $0.25: 1$. The polymerisation was done in stop flow for $20 \mathrm{~h}$. This molar ratio was chosen as it has been previously shown that polymerisation of aniline in these experimental conditions produces nanofibres[28]. After polymerisation, the channels were washed extensively with water and ethanol to remove any unattached polyaniline nanofibres. The resulting coating was characterised by SEM showing that the PAni coating was obtained in the nanofibres form (see ESI, Fig. S1) with good adhesion due to the covalent bonding between the substrate (PDMS, glass) and polymer coating.

\subsection{Experimental detection of the mixing point}

For experimental recording of the mixing point, colourless solutions of hydrochloric acid $\left(10^{-}\right.$ $\left.{ }^{2} \mathrm{M}, \mathrm{pH}=2\right)$ and sodium hydroxide $\left(10^{-2} \mathrm{M}, \mathrm{pH}=12\right)$ were pumped into the two arms of a Y-shaped microchannel at the respective equal flow rates, which were varied between $1 \mu \mathrm{L}$ $\min ^{-1}$ to $10.5 \mu \mathrm{L} \mathrm{min}{ }^{-1}$ in $0.5 \mu \mathrm{L} \mathrm{min}{ }^{-1}$ increments. A replica of the microfluidic channel was cut in PSA containing mm marks parallel to the channel, and laminated to the bottom glass layer of the PAni coated microfluidic chip. A series of images were taken using an Aigo GE5 microscope using an 180x objective lens with the accompanying software. Following this, the mixing point was recorded as the point where the green colour of the PAni coating specific to the acidic solution pumped through the channel disappeared. The solutions were 
pumped through the channels at the respective flow rates for at least $15 \mathrm{~min}$ with no substantial variation in the mixing point observed.

\subsection{Numerical solver}

A finite volume method (FVM) based numerical fluid flow solution methodology [29] was used to obtain decoupled solution for both the Navier-Stokes (NS) equations and the mass diffusion equations (convection-diffusion equations) in the absence of reaction-transport equations and involving a high degree of convection-diffusion discretisation schemes, applied to solve the combined effects of both convection and diffusion.

A segregated steady flow solver (pressure based segregated algorithm) was used. For discretisation the PRESTO (pressure staggering options) scheme was used for pressure interpolation, the SIMPLE (Semi-Implicit Method for Pressure-Linked Equations) scheme was used for pressure-velocity coupling, and the second-order up-wind differencing scheme was used for the momentum (fluid flow) and species transport equations. All fluid properties - including density and viscosity were assumed to be constant for all simulations, which were conducted under laminar flow assumption, as the flow in the microchannel was typically laminar because of the very low Reynolds number of the present system (typically less than 1). The convergence criterion was also set to $10^{-16}$ for the residuals of velocities, species, and continuity to attain high numerical accuracy.

\subsection{Governing flow equations}

The fluid flow was considered to be steady, viscous, incompressible, Newtonian, i.e. the present fluid maintained constant density and viscosity during the mixing and diffusion processes with no-change of temperature, and three-dimensional (3-D) with assumption of 
laminar flow behaviour at very low Reynolds number range as the viscous forces highly predominate the inertia forces.

The energy conservation equation is not discretised under isothermal flow conditions and the governing flow equations are conservation equations for mass and momentum. The mass conservation or continuity equation is then described as:

$$
\nabla(\vec{v})=0
$$

where $\vec{v}$ is velocity vector field, and the equation is applicable for the present $3-\mathrm{D}$, steady and incompressible flow. The differential form of the equations for the conservation of momentum of viscous fluids is obtained by applying Newton's second law of motion to an infinitesimal fluid element and the resultant momentum equation for an inertial reference frame in conservation form, by neglecting the curvature and surface tension due to predominant viscous forces, is described here as follows:

$$
\nabla(\vec{v} \vec{v})=-\frac{1}{\rho} \nabla p+\nabla(\bar{\tau})+\vec{g}+\vec{f}
$$

where, $p$ is the static pressure, $\rho$ is the fluid density, $\bar{\tau}$ is the stress tensor (as described below), $\vec{g}$ and $\vec{f}$ are the gravitational vector and external body force per unit mass, respectively. The stress tensor is expressed as:

$$
\bar{\tau}=\mu\left[\left(\nabla \vec{v}+\nabla \vec{v}^{T}\right)-\frac{2}{3} \nabla \vec{v} I\right]
$$

Where, $\mu$ is the molecular viscosity, and $I$ is the unit tensor. For the steady, incompressible fluid flow of microfluidic systems, local time derivative terms are neglected and the above Navier-Stokes equations are drastically simplified as the ratio of the inertia terms to the viscous term (characterised by the Reynolds number $\operatorname{Re})$ becomes negligible $(\operatorname{Re}<<1)$. In addition to conservation equations for mass and momentum, fluid transport equations in binary mixtures in absence of temperature or pressure gradient can be described as[29]: 


$$
J_{i}=-\rho\left(\sum_{j=1}^{n-1} D_{i j} \nabla c_{i}\right)
$$

Where $D_{i j}$ is the molecular diffusion coefficient, $c_{\mathrm{i}}$ is the mass fraction and $J_{i}$ is the flux of the $i$ analyte of interest for $n$ number of components. Since turbulence does not occur for such problem, fluid mixing mainly occurs at the microscale due to molecular diffusion mechanism at the interface between two fluids. Therefore, species diffusion mechanism was modelled with use of the species transport modelling approach accompanied with "moleculardiffusion" option. For quantification of the degree of mixing into the present microchannel, the following below referenced equation was also used, as described by Lu et al.[30].

$$
\text { Degree of mixing }=\left[1-\frac{\sqrt{\frac{\sum_{i=1}^{N}\left(c_{i}-\bar{c}\right)^{2}}{N}}}{\bar{c}}\right] \times 100
$$

Where $\bar{c}$ is the average mass fraction of the mixture and $c_{i}$ is the mass fraction of the analyte of interest in the ith cell and $N$ is the total number of cells in the cross-section. The degree of mixing was calculated at different cross-sections along the mixing channel. The mixing efficiency ranged from 0 to $100 \%$ efficiency.

\subsection{Computational domain and boundary conditions.}

The microchannel geometry, which was accompanied with Y-junction inlet where the two fluid steams met, was built by the 3-D geometry modular in CATIA programme and had a total length of $40 \mathrm{~mm}$ in the $y$-direction, $15.9 \mathrm{~mm}$ in the $x$ direction, and a gap of $0.18 \mathrm{~mm}$ in the $z$ direction where the gravitational effects were considered.

The computational domain of the microchannel was constructed of non-uniformly spaced structured hexahedral finite volume cells using a pre-processor module [31]. The initial mesh 
independency study was conducted starting with the number of 320,500 hexahedral cells, which were gradually increased up to 812,500 cells, with most of the volume cells having all sides about $40 \mu \mathrm{m} \times 18 \mu \mathrm{m}$. The flow domain had 25 cells in the $-x$ direction and 10 cells in in the $-z$ direction (the span-wise direction) for the final mesh configuration.

Figure 1a illustrates the 2-D representation of the 3-D computational flow domain of the microfluidic system on the $-x y$ plane while Figure $1 \mathrm{~b}$ and $\mathrm{c}$ represent the $3-\mathrm{D}$ local mesh in the $y$-branch and the 2-D grid layout on the $-x z$ plane together, respectively for the final mesh configuration of 812,500 hexahedral cells.

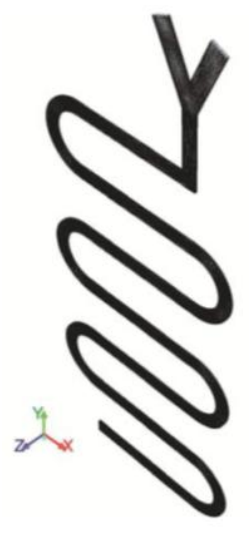

(a)

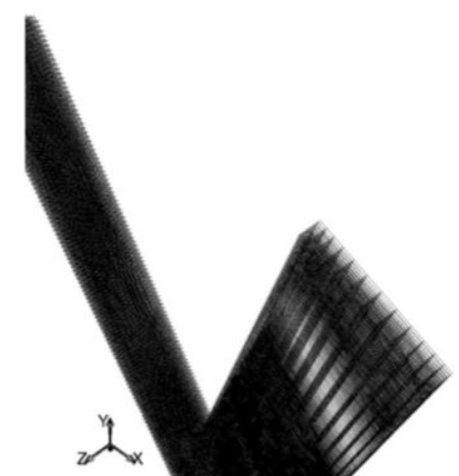

(b)

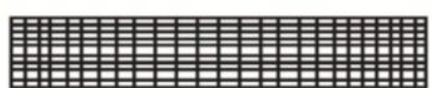

(c)

Figure 1. Three-dimensional (3-D) computational flow domain of the microfluidic system a) 2-D representation on the $-x y$ plane b) 3-D local mesh in the $y$-branch c) 2-D grid layout on the $-x z$ plane.

The numerical simulations were performed in 3-D manner based on the assumption of steady, incompressible and isothermal fluid flow conditions, using a laminar modelling approach by considering the following boundary conditions:

1. Velocity inlet boundary conditions: Velocity inlet boundary conditions were used to define the fluid stream velocities and mass fraction of the species of interests at the inlet boundary. The net transport of species at the inlet consisted of both convection and diffusion 
components. The convection component was specified with mass concentration rate of 0.375 $\%$ and $0.4 \%$ for $\mathrm{HCl}$ and $\mathrm{NaOH}$ fluid streams, respectively. The diffusion component, however, was not specified a priori as it depended on the gradient of the computed species at the inlet. The specified inlet velocity for each fluid stream also ranged from $1 \mu \mathrm{L} \mathrm{min}{ }^{-1}$ to $10.5 \mu \mathrm{L} \mathrm{min}{ }^{-1}$ at each branch of the $\mathrm{Y}$-junction.

2. Outlet Boundary Conditions: Atmospheric pressure outlet boundary condition was applied to the outlet of the geometry, allowing the outflow value of pressure to atmospheric.

3. No-slip boundary condition: No-slip boundary condition was imposed at the rigid wall boundary which was assumed to be an inert material with zero mass flux normal to the wall surface.

The properties of the mixtures introduced into the device are summarised in Table 1 below.

Table 1. Properties of the mixture introduced into the microchannel [24].

\begin{tabular}{cc}
\hline \multicolumn{3}{c}{$\mathrm{HCl}-\mathrm{H}_{2} \mathrm{O} \mathrm{T}_{0}=298.16 \mathrm{~K}$} \\
\hline$c_{\text {inlet }}(\%)$ & $D_{(\mathrm{HCl})}\left(\mathrm{m}^{2} / \mathrm{s}\right)$ \\
\hline 0.375 & $8.7 \times 10^{-9}$ \\
\hline \multicolumn{3}{c}{${\mathrm{NaOH}-\mathrm{H}_{2} \mathrm{O} \mathrm{T}_{0}=298.16 \mathrm{~K}} D_{(\mathrm{NaOH})}\left(\mathrm{m}^{2} / \mathrm{s}\right)$} \\
\hline$c_{\text {inlet }}(\%)$ & $1.33 \times 10^{-9}$ \\
\hline 0.4 &
\end{tabular}

The density and viscosity were both assumed to be constant in the simulations since the variations in $\mathrm{HCl}$ and $\mathrm{NaOH}$ concentration during the experiments reflected a negligible change in viscosity and density values, being the value of the pure water $\left(\rho=998,4 \mathrm{Kg} \mathrm{m}^{-3}\right.$; $\mu=0,001 \mathrm{Kg} \mathrm{m}^{-1} \mathrm{~s}^{-1}$ ). Taking into account the high degree of complexity of analysing such multi-component mixing $\left(\mathrm{HCl}-\mathrm{NaOH}-\mathrm{H}_{2} \mathrm{O}\right)$ process, further simplifications were required. The mass flux in a multicomponent system was represented due to[32]:

$$
J_{1}=-\rho D_{11} \frac{\partial c_{1}}{\partial x}-\rho D_{12} \frac{\partial c_{2}}{\partial x}
$$




$$
J_{2}=-\rho D_{21} \frac{\partial c_{1}}{\partial x}-\rho D_{22} \frac{\partial c_{2}}{\partial x}
$$

Where 1, 2 and 3 correspond to the different analytes, $J_{1}, J_{2}$ and $J_{3}$ which are the flux of each analite in the $x$ diffusion direction where $J_{1}+J_{2}+J_{3}=0$, and $c_{1}+c_{2}+c_{3}=1$. As crossmolecular diffusion coefficients, $D_{12}$ and $D_{21}$ in the above equation are two orders of magnitude smaller than direct diffusion coefficients, $D_{11}$ or $D_{22}$, they were assumed to be negligible[33].

\section{Results and discussion}

\subsection{Characterisation of the PAni coatings}

Using the technique described in the experimental section homogeneous PAni coatings were obtained on the microchannel surface while maintaining the nanomorphology of PAni, as previously reported[17]. The main advantages of having nanofibres (versus bulk PAni) is the high surface area which in turn ensures short diffusional path lengths[34] enabling improved sensitivity and short response times[35-37]. Our previous studies have showed that when different $\mathrm{pH}$ solutions are passed through the PAni coated fluidics, at similar flow rates with what is described here, these PAni coatings show a fast response time and a fast regeneration time of less than $5 \mathrm{~s}$ at room temperature [18].

The PAni coatings were characterised by UV-Vis spectroscopy as it permits in-situ optical analysis of PAni coating inside the microchannel (see ESI $\dagger$ - Figure S2 for the experimental set-up). These PAni coatings respond very well to changes in $\mathrm{pH}$ as shown by the absorption measurements of the channel coating, Figure S3. When a solution of $\mathrm{pH} 2$ is passed through the microchannel, PAni has a green colour and presents the typical bands for the emeraldine salt (ES), showing a characteristic absorption band at around $360 \mathrm{~nm}$ associated with $\pi-\pi^{*}$ transition of the conjugated ring system[38] and an absorption band at $\sim 420 \mathrm{~nm}$ together with an extended tail at $850 \mathrm{~nm}$ assigned to a polaron band transition[38]. When a solution of $\mathrm{pH}$ 
11 is flushed through the microchannel, the PAni coating changes its colour from green to blue, reflecting formation of the dedoped emeraldine base (EB) state, see Figure 2. The band at $610 \mathrm{~nm}$ is ascribed to exciton formation in the quinonoid rings and this absorption gives rise to the blue colour of the PAni coatings[38].

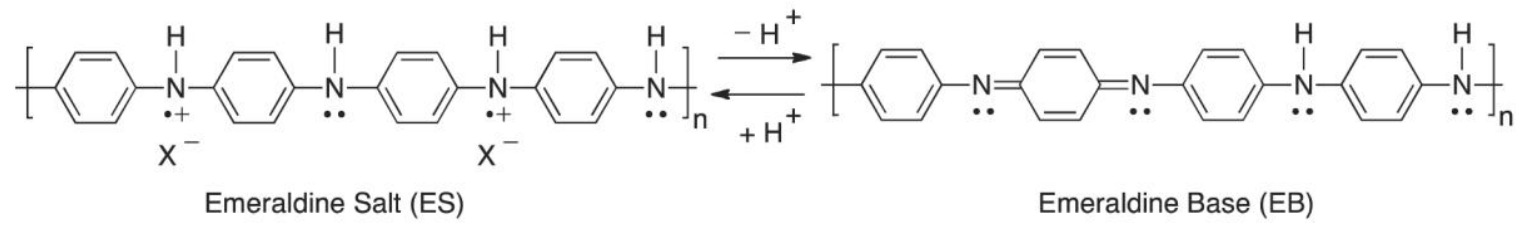

Figure 2. Protonated (doped) polyaniline emeraldine salt (ES) is deprotonated (dedoped) by treatment with an alkali to polyaniline emeraldine base (EB).

To study diffusion and mixing in this device, colourless hydrochloric acid $\left(10^{-2} \mathrm{M}, \mathrm{pH}=2\right)$ and sodium hydroxide $\left(10^{-2} \mathrm{M}, \mathrm{pH}=12\right)$ solutions were pumped into the two arms of a $\mathrm{Y}$ shaped microchannel, $1000 \mu \mathrm{m}$ in width and $180 \mu \mathrm{m}$ in height (Figure 3). The two liquid streams meet at the Y-junction, and have an interaction time defined by the flow rate, which was varied between $1 \mu \mathrm{L} \min ^{-1}$ to $10.5 \mu \mathrm{L} \mathrm{m^{-1 }}$. As upon dedoping the colour of the PAni coating becomes blue, the mixing point is represented by the disappearance of the green colour, specific to the doped form of PAni, ES. A plot of the mixing point (i.e. the point at which the green colour disappears relative to the meeting point at the Y-junction) against flow rate shows the evolution of the mixing point as a function of flow rate (Figure 4).

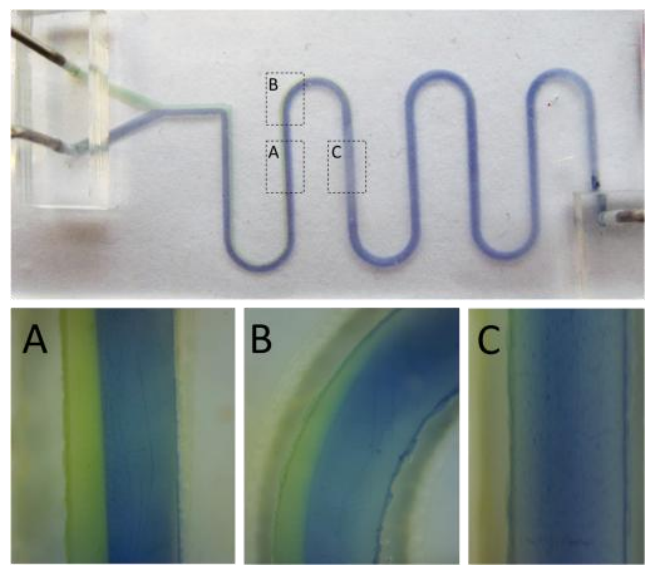


Figure 3. Photo showing the PAni functionalised Y-shaped microfluidic device when colourless solutions of $\mathrm{HCl}$ and $\mathrm{NaOH}$ were pumped into the two opposite arms of the channel. Insets display microscope images showing the colour of the PAni coatings during the mixing of the two laminar flows $(\mathrm{HCl}$ and $\mathrm{NaOH})$ inside the channel at different positions $(A, B, C)$.

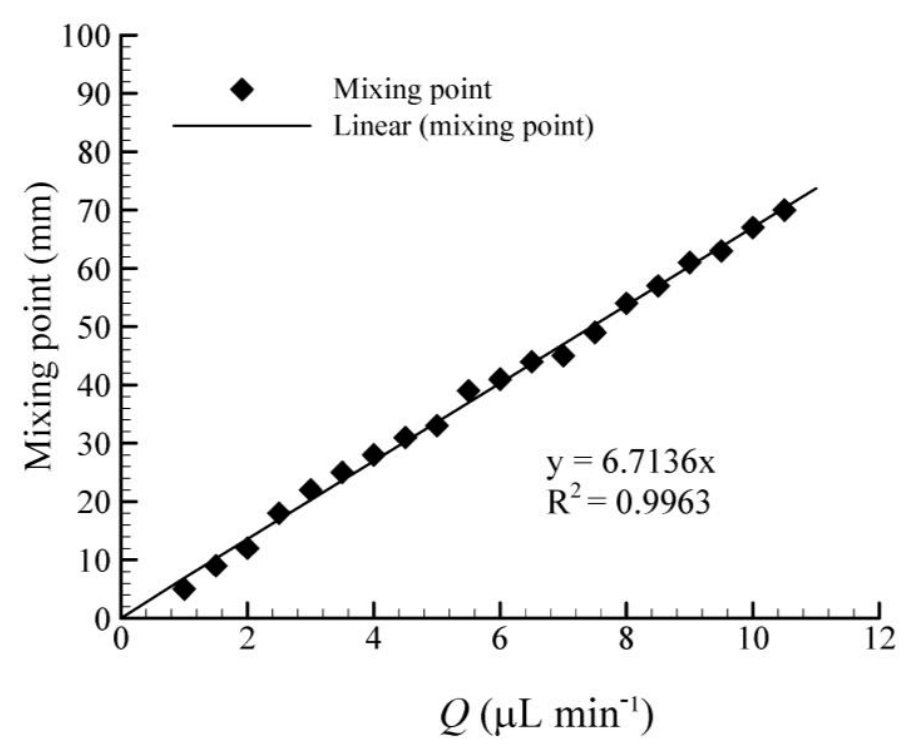

Figure 4. Graph showing the evolution of the experimentally recorded mixing point as a function of flow rate, $Q$.

\subsection{Computational results}

In order to verify the experimental results, computational results were carried out as described in the Experimental section. In a diffusive mixing process, the mixing velocity is highly dependent on the concentration gradient of the components, and concentration gradient(s) drastically reduces at very higher values of mixing degree(s). Therefore, it is nearly impossible to reach $100 \%$ mixing degree for a finite length of microfluidic device. Bearing this in mind, in the present study, the concentration degree of $99.5 \%$ was considered to be a threshold point at which the full mixing could be obtained. From this point onward, 
the change in the concentration rate was almost negligible (as it reached its asymptotic value).

For better quantifying the mixing effects in the microfluidic flow system, a dimensionless number called the Péclet number $(\mathrm{Pe})$, which is the ratio of the contributions to mass transport by convection to those by diffusion:

$$
P e=\frac{N_{\text {conv }}}{N_{\text {diff }}}=\frac{c_{i}|V|}{D \nabla c_{i}}=\frac{L V}{D}
$$

where $L$ is a characteristic length scale, $V$ is the velocity magnitude, and $D$ is a characteristic diffusion coefficient is used. The Péclet number for mass transport is comparable to the Reynolds number for momentum transport. Because the Péclet number is proportional to system size, it is found that at small scales, diffusion contributes much more effectively to mass transfer, so mixing can be achieved without stirring.

Figure 5 below illustrates the computed degree of mixing for the inflow volumetric flow rate ranging from $1 \mu \mathrm{L} \mathrm{min}{ }^{-1}$ to $10.5 \mu \mathrm{L} \mathrm{min}{ }^{-1}$, with the corresponding Peclet number ranging from 139.24 to 696.18 , respectively. The Peclet number clearly affects the degree of mixing, which increases up to an asymptotic value of $100 \%$ for each specified inflow velocity value. The higher the inflow velocity, i.e. Peclet number, the higher the mixing length is required to reach the full mixing. The evolution of the mixing degree with the Peclet number correlated well with the analytical data [39]. 


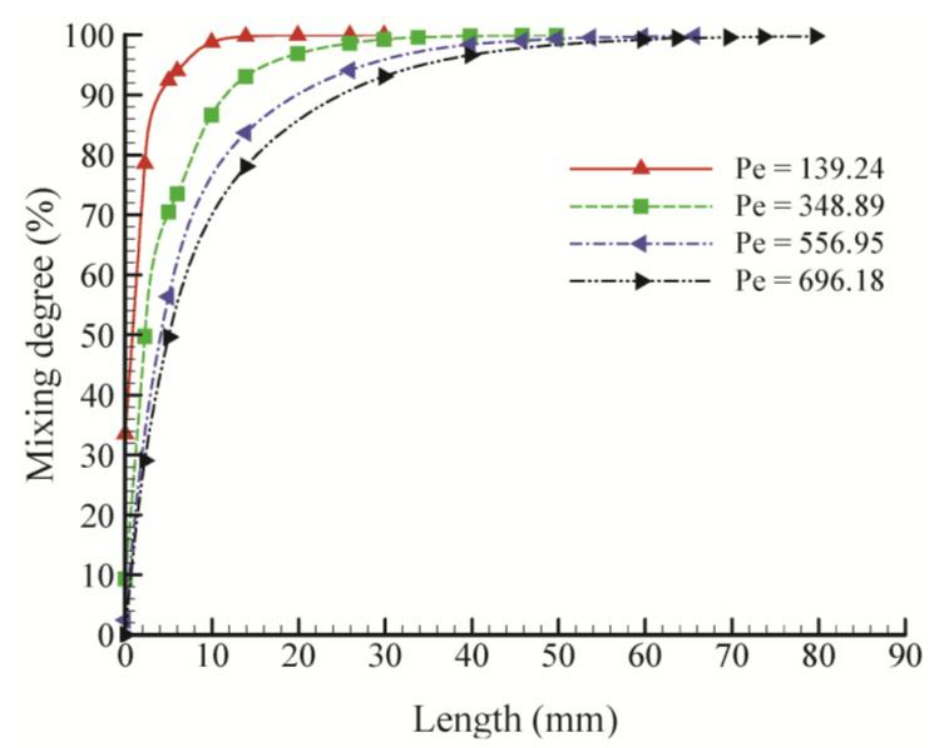

Figure 5. Mixing degree vs. channel length at different Peclet numbers.

A plot of the mixing point versus flow rate varied over 10 different inflow velocity values is depicted in Figure 6 using Peclet number values. Both, numerical and experimental values present good linearity demonstrating the efficiency of the adopted numerical approach for the investigation of mixing and molecular diffusion in the microchannel when compared with the experimental data. The numerical results obtained from the final configuration of 812,500 hexahedral cells correspond well with the experimental data with a maximum error of $4 \%$ at a lowest inflow velocity of $1 \mu \mathrm{L} \mathrm{min}{ }^{-1}$.

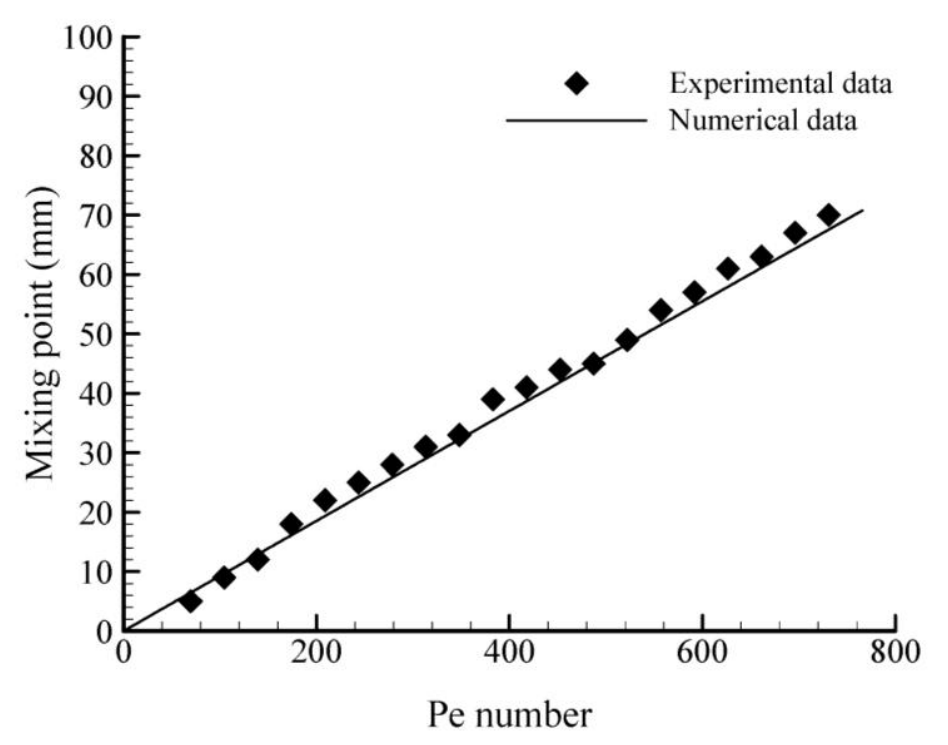


Figure 6. Mixing point at different Peclet numbers for both, experimental visualisation results (black diamonds) and for the numerical modelling results (straight line).

In the present study, to find the mixing point, a simplified analytical approximation that can be used in binary mixtures is adopted with the numerical modelling approach. According to this approximation, the linear relation between the mixing point and Reynolds number leads to a below diffusion time expression as[39].

$$
t_{d i f f}=\frac{L^{2}}{2 D}
$$

where $L$ is the diffusion length i.e. the mean width of the channel and $D$ is the diffusion coefficient. The residence time can be also estimated within this approximation accompanied with the numerical modelling approach as:

$$
t_{\text {residence }}=\frac{L_{\text {residence }}}{\bar{V}}
$$

where $\bar{V}$ is the mean velocity ( $=Q / L^{2}$, where $Q$ is the flow rate, and $L$ is the characteristic dimension of the microfluidic device), and $L_{\text {residence }}$ is the necessary length in the convective flow direction for the total mixing. Moreover, it is necessary that the diffusion time is higher than the residence time $t_{\text {diff }}>t_{\text {residence }}$, indicating that:

$$
L_{\text {residence }}>\frac{L^{2}}{2 D} \bar{V}
$$

with following below expressions:

$$
P e=\operatorname{Re} S c=\frac{L \bar{V}}{D}
$$

and

$$
L_{\text {residence }}>\frac{\operatorname{Re} S c L}{2}
$$


where $L$ is the width of the channel. Consequently the mixing point distance has a linear relation with Reynolds number as $\frac{L_{\text {residence }}}{\operatorname{Re}}>$ constant value, which is approximated to $\frac{S c L}{2}$ $\approx$ constant here.

The qualitative results obtained from the numerical results are also represented in terms of mass fraction contours obtained at different 2-D planes- cross-sections for two different inflow flow rates of $2 \mu \mathrm{L} \mathrm{min}{ }^{-1}$ and $10 \mu \mathrm{L} \mathrm{min}^{-1}$ in the direction of the flow. Figure 7 clearly shows the evolution of mixing and diffusion processes as the acid and alkali from the two sources progress through the microfluidic system, and visualises how the shape of the interface between the fluids changes as the flow progress in the downstream direction. In each zone, the mixing zone is confined to a narrow band around the interface of two fluids, and the band becomes narrower as the degree of mixing increases. Therefore, the numerical approach can qualitatively identify the mixing behaviour in a very realistic manner and provides a means to predict experimental conditions under which a particular mixing regime will exist for a particular microfluidic system design.

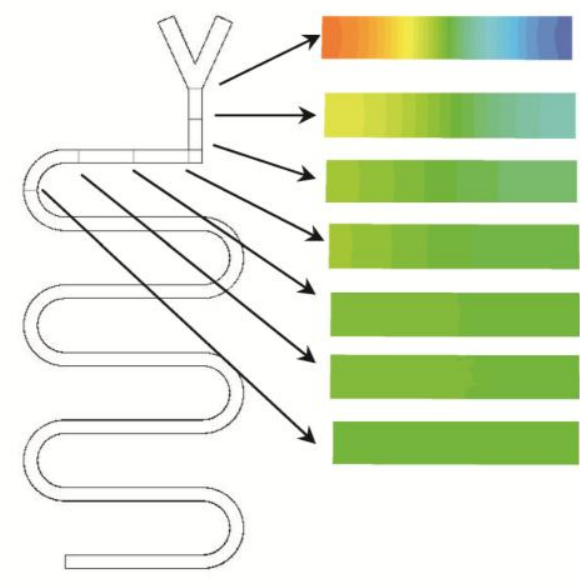

(a)

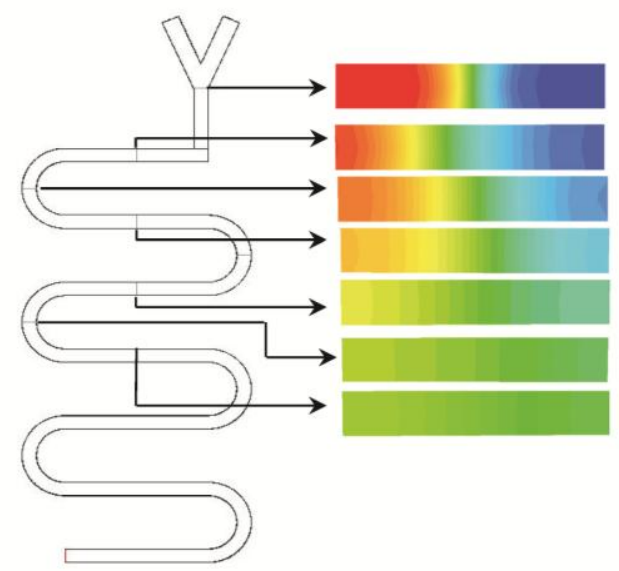

(b)

Figure 7. Representation of the mixing behaviour in terms of mass fraction contours for cases a) $Q=2 \mu \mathrm{L} \mathrm{min}{ }^{-1}$; and b) $Q=10 \mu \mathrm{L} \min ^{-1}$. Red represents the $\mathrm{NaOH}$ solution and blue the $\mathrm{HCl}$ solution; when mixing is complete colour becomes light green. 


\section{Conclusions}

The point of complete mixing and diffusion dynamics of two reacting solutions exemplified by $\mathrm{HCl}$ and $\mathrm{NaOH}$ in a glass/PDMS microfluidic device can be directly visualised using adaptive coatings based on the conductive polymer, polyaniline (PAni). The experimental results are in excellent agreement with numerical modelling approaches.

The present computational modelling approach validated with the experimental data form a basis for qualitatively and quantitatively determining the flow parameter variations and possible design challenges to optimise the performance of microfluidic devices. Since this technique is based on $\mathrm{pH}$ responsive coatings, at present, it is not suitable for mixing evaluation in 3D but rather 2D (along the length and width of the channel) and as such can not provide information on the $\mathrm{pH}$ generated gradients inside the channel as a consequence of diffusion of the two laminar flows (exemplified here by $\mathrm{HCl}$ and $\mathrm{NaOH}$ ). Nevertheless, PAni coatings still offer valuable information for the point of complete mixing as supported by the numerical modeling data. The validated model therefore could be a useful design tool for development and optimisation of microchannel design and operational parameters (channel dimensions, geometry, surface roughness, flow rates etc.) for applications in which diffusion control is essential.

\section{Conflict of interest statement}

There is no conflict of interest about this article.

\section{Acknowledgements}

The project was carried out with the support of Science Foundation Ireland under INSIGHT award $\mathrm{SFI} / 12 / \mathrm{RC} / 2289$. This work was supported by Gobierno Vasco, Dpto. Industria, Innovación, Comercio y Turismo under ETORTEK 2013 and ETORTEK 2014 for the project undertaken by 
Microchip and the project of Fluids Mechanics Groups (IT557-10) of Gobierno Vasco, Dpto. de Educación. F.B.L. acknowledges the Ramón y Cajal programme (Ministerio de Economía y Competitividad). FBL personally acknowledges Marian M. De Pancorbo for letting him to use her laboratory facilities at UPV/EHU.

\section{Appendix A. Supplementary data}

Supplementary data associated with this article can be found, in the online version, at

\section{Vitae}

Include in the manuscript a short (maximum 100 words) biography of each author.

Larisa Florea studied organic chemistry and chemical engineering at University "Politehnica" from Timisoara, Romania (B.Sc. Hons 2009). In 2009 she joined the Adaptive Sensors Group at Dublin City University where she earned her Ph.D. degree under the supervision of Prof. Dermot Diamond and Dr. Fernando Benito-Lopez. Her research interests include the development of stimuli-responsive polymers as novel sensing materials in microfluidics.

Alain Martin received his $\mathrm{Ph}$. D degree in mechanical engineering from the University of Mondragon, Spain in 2012. He is currently working as teacher and researcher at the same university. His research interest includes the computational modeling and experimentation of separation and mixture processes in multiphase fluxes.

M. Mounir Bou-Ali received his Ph. D in Physics (UPV / EHU, 1999). He has developed his research during his research in the areas of Fluid Mechanics and transport phenomena. Since 2002 he has been teaching at the University of Mondragon, with research in fluid mechanics activity, being the coordinator of the line of R \& D in Fluid Mechanics. He is currently developing his research on issues related to transport phenomena in liquid mixtures; synthesis, characterization and analysis of magnetorheological fluids, as well as the development of new methods of separating in micro-biotechnological. He has published over 
150 papers in scientific journals, book chapters, international and national conferences and has made 10 patents.

Kate Meagher studied Analytics Science at Dublin City University, Ireland. In 2012, she obtained her B. Sc. degree in chemistry and performed a research project on "Polyaniline nanofibres for sensing applications" under the supervision of Prof. Dermot Diamond and Dr. Fernando Benito-Lopez.

Dermot Diamond received his Ph.D. and D.Sc. from Queen's University Belfast (Chemical Sensors, 1987, Internet Scale Sensing, 2002), and was Vice-President for Research at Dublin City University (2002-2004). He has published over 300 peer-reviewed papers in international journals, is a named inventor in 18 patents, and is co-author and editor of four books. He is director and founding member of the National Centre for Sensor Research (www.ncsr.ie) at Dublin City University, and an SFI-funded investigator in the INSIGHT Centre (http://www.insight-centre.org).

Mustafa Tutar received his Ph.D. degree in computational fluid dynamics (CFD) from the University of Hertfordshire, UK in 1998. He is currently working as IKERBASQUE research professor at the University of Mondragon in Spain. His research interests include computational modeling of fluid-structure interactions and mathematical modeling of turbulent flow and heat transfer for different flow scenarios.

Fernando Benito-Lopez studied chemistry at the Universidad Autonoma de Madrid and completed his master studies in the Department of Inorganic Chemistry in 2002. He obtained his $\mathrm{PhD}$ at the University of Twente, The Netherlands, in 2007. He carried out his postdoctoral research in the group of Prof. Dermot Diamond at Dublin City University, Dublin, where in 2010, he became Team Leader in polymer microfluidics. In 2012 he moved to CIC microGUNE a Research Centre working in Microtechnology in Spain. From 2015 he is Ramón y Cajal Fellow at the University of the Basque Country, Spain. 


\section{REFERENCES}

[1] K.D. Dorfman, M. Chabert, J.-H. Codarbox, G. Rousseau, P. de Cremoux, J.-L. Viovy, Contamination-free continuous flow microfluidic polymerase chain reaction for quantitative and clinical applications, Anal Chem, 77(2005) 3700-4.

[2] L. Capretto, W. Cheng, M. Hill, X. Zhang, Micromixing within microfluidic devices, Microfluidics, Springer2011, pp. 27-68.

[3] Z. Yang, S. Matsumoto, H. Goto, M. Matsumoto, R. Maeda, Ultrasonic micromixer for microfluidic systems, Sensors and Actuators A: Physical, 93(2001) 266-72.

[4] H.H. Bau, J. Zhong, M. Yi, A minute magneto hydro dynamic (MHD) mixer, Sens Actuators B: Chem, 79(2001) 207-15.

[5] A.D. Stroock, S.K.W. Dertinger, A. Ajdari, I. Mezic, H.A. Stone, G.M. Whitesides, Chaotic mixer for microchannels, Science, 295(2002) 647-51.

[6] R.H. Liu, M.A. Stremler, K.V. Sharp, M.G. Olsen, J.G. Santiago, R.J. Adrian, et al., Passive mixing in a three-dimensional serpentine microchannel, Microelectromechanical Systems, Journal of, 9(2000) 190-7.

[7] G. Ocvirk, T. Tang, D.J. Harrison, Optimization of confocal epifluorescence microscopy for microchip-based miniaturized total analysis systems, Analyst, 123(1998) 1429-34.

[8] J.E. Melanson, C.A. Lucy, Violet (405 nm) diode laser for laser induced fluorescence detection in capillary electrophoresis, Analyst, 125(2000) 1049-52.

[9] K. Shinohara, Y. Sugii, A. Hibara, M. Tokeshi, T. Kitamori, K. Okamoto, Rapid proton diffusion in microfluidic devices by means of micro-LIF technique, Exp Fluids, 38(2005) 117-22.

[10] T. Park, M. Lee, J. Choo, Y.S. Kim, E.K. Lee, D.J. Kim, et al., Analysis of passive mixing behavior in a poly(dimethylsiloxane) microfluidic channel using confocal fluorescence and Raman microscopy, Appl Spectrosc, 58(2004) 1172-9.

[11] H. Salimi-Moosavi, Y.T. Jiang, L. Lester, G. McKinnon, D.J. Harrison, A multireflection cell for enhanced absorbance detection in microchip-based capillary electrophoresis devices, Electrophoresis, 21(2000) 1291-9.

[12] H. Lu, M.A. Schmidt, K.F. Jensen, Photochemical reactions and on-line UV detection in microfabricated reactors, Lab Chip, 1(2001) 22-8.

[13] G.M. Greenway, L.J. Nelstrop, S.N. Port, Tris(2,2-bipyridyl)ruthenium (II) chemiluminescence in a microflow injection system for codeine determination, Anal Chim Acta, 405(2000) 43-50.

[14] Y. Xu, F.G. Bessoth, J.C.T. Eijkel, A. Manz, On-line monitoring of chromium(III) using a fast micromachined mixer/reactor and chemiluminescence detection, Analyst, 125(2000) 677-83.

[15] A.B. Shrirao, R. Perez-Castillejos, Simple Fabrication of Microfluidic Devices by Replicating Scotch-tape Masters, Chips \& Tips, (17 May 2010).

[16] B.H. Weigl, R.L. Bardell, N. Kesler, C.J. Morris, Lab-on-a-Chip sample preparation using laminar fluid diffusion interfaces-computational fluid dynamics model results and fluidic verification experiments, Fresenius' journal of analytical chemistry, 371(2001) 97105.

[17] L. Florea, C. Fay, E. Lahiff, T. Phelan, N.E. O'Connor, B. Corcoran, et al., Dynamic pH mapping in microfluidic devices by integrating adaptive coatings based on polyaniline with colorimetric imaging techniques, Lab Chip, 13(2013) 1079-85.

[18] L. Florea, D. Diamond, F. Benito-Lopez, Polyaniline coated micro-capillaries for continuous flow analysis of aqueous solutions, Anal Chim Acta, 759(2013) 1-7. 
[19] L. Castelletti, S.A. Piletsky, A.P.F. Turner, P.G. Righetti, A. Bossi, Development of an integrated capillary electrophoresis/sensor for L-ascorbic acid detection, Electrophoresis, 23(2002) 209-14.

[20] L. Florea, A. McKeon, D. Diamond, F. Benito-Lopez, Spiropyran polymeric microcapillary coatings for photodetection of solvent polarity, Langmuir, 29(2013) 2790-7.

[21] F. Benito-Lopez, S. Scarmagnani, Z. Walsh, B. Paull, M. Macka, D. Diamond, Spiropyran modified micro-fluidic chip channels as photonically controlled self-indicating system for metal ion accumulation and release, Sens Actuators, B, 140(2009) 295-303.

[22] H. Hisamoto, Y. Nakashima, C. Kitamura, S. Funano, M. Yasuoka, K. Morishima, et al., Capillary-assembled microchip for universal integration of various chemical functions onto a single microfluidic device, Anal Chem, 76(2004) 3222-8.

[23] S. Stafström, J. Bredas, A. Epstein, H. Woo, D. Tanner, W. Huang, et al., Polaron lattice in highly conducting polyaniline: theoretical and optical studies, Phys Rev Lett, 59(1987) 1464.

[24] E. Pringsheim, E. Terpetschnig, O.S. Wolfbeis, Optical sensing of $\mathrm{pH}$ using thin films of substituted polyanilines, Anal Chim Acta, 357(1997) 247-52.

[25] Z. Jin, Y.X. Su, Y.X. Duan, An improved optical pH sensor based on polyaniline, Sens Actuators, B, 71(2000) 118-22.

[26] U.W. Grummt, A. Pron, M. Zagorska, S. Lefrant, Polyaniline based optical pH sensor, Anal Chim Acta, 357(1997) 253-9.

[27] C.-G. Wu, J.-Y. Chen, Chemical deposition of ordered conducting polyaniline film via molecular self-assembly, Chemistry of materials, 9(1997) 399-402.

[28] J.F. Qiang, Z.H. Yu, H.C. Wu, D.Q. Yun, Polyaniline nanofibers synthesized by rapid mixing polymerization, Synth Met, 158(2008) 544-7.

[29] K. Ghorayeb, A. Firoozabadi, Modeling multicomponent diffusion and convection in porous media, SPE Journal, 5(2000) 158-71.

[30] L.-H. Lu, K.S. Ryu, C. Liu, A magnetic microstirrer and array for microfluidic mixing, Microelectromechanical Systems, Journal of, 11(2002) 462-9.

[31] N. Fluent Dynamics Software. ANSYS FLUENT. Lebanon, (2010).

[32] M. Larrañaga, D.A.S. Rees, M.M. Bou-Ali, Determination of the molecular diffusion coefficients in ternary mixtures by the sliding symmetric tubes technique, The Journal of chemical physics, 140(2014) 054201.

[33] M. Bou-Ali, A. Ahadi, D.A. de Mezquia, Q. Galand, M. Gebhardt, O. Khlybov, et al., Benchmark values for the Soret, thermodiffusion and molecular diffusion coefficients of the ternary mixture tetralin+ isobutylbenzene+ n-dodecane with 0.8-0.1-0.1 mass fraction, The European Physical Journal E, 38(2015) 1-6.

[34] J.X. Huang, Syntheses and applications of conducting polymer polyaniline nanofibers, Pure and Applied Chemistry, 78(2006) 15-27.

[35] J.X. Huang, S. Virji, B.H. Weiller, R.B. Kaner, Polyaniline nanofibers: Facile synthesis and chemical sensors, J Am Chem Soc, 125(2003) 314-5.

[36] J. Huang, S. Virji, B.H. Weiller, R.B. Kaner, Nanostructured polyaniline sensors, Chemistry-a European Journal, 10(2004) 1315-9.

[37] S. Virji, J.D. Fowler, C.O. Baker, J.X. Huang, R.B. Kaner, B.H. Weiller, Polyaniline manofiber composites with metal salts: Chemical sensors for hydrogen sulfide, Small, 1(2005) 624-7.

[38] G.G. Wallace, G.M. Spinks, L.A.P. Kane-Maguire, P.R. Teasdale, Conductive electroactive polymers: CRC Press LLC; 2003.

[39] A. Einstein, Investigations on the Theory of the Brownian Movement: Courier Corporation; 1956. 
